Trauma Berufskrankh 2016 · [Suppl 3]:

18:S222-S225

DOI 10.1007/s10039-015-0069-6

Online publiziert: 21. Juli 2015

(c) Springer-Verlag Berlin Heidelberg 2015

CrossMark
M. Walther · A. Röser

Schön Klinik München Harlaching, München, Deutschland

\section{Therapie der posttraumatischen Arthrose am Sprunggelenk}

\section{Argumente für eine Arthrodese}

Die Prävalenz der Arthrose am oberen Sprunggelenk wird bei der erwachsenen Bevölkerung mit ca. $1 \%$ angegeben [10]. In $56 \%$ der Fälle entwickelte sich die Arthrose infolge eines Sprunggelenktraumas [2]. Als konservative Therapieoptionen stehen Injektionen, stabilisierende Orthesen bzw. die Versorgung mit einem das Sprunggelenk umfassenden orthopädischen Schuh zur Verfügung. Die Arthrodese und die endoprothetische Versorgung des oberen Sprunggelenks werden bis heute kontrovers diskutiert. Trotz der zweifelsohne während der letzten Jahre vorhandenen Erfolge der Endoprothetik liegen die Ergebnisse unverändert deutlich hinter denen, die beispielsweise in der Hüftendoprothetik erreicht werden.

\section{Endoprothetik}

Bei einem oberflächlichen Blick in die aktuelle Literatur entsteht der Eindruck, dass zwischenzeitlich die meisten Probleme, die mit früheren Generationen der Sprunggelenkendoprothese vorhanden waren, gelöst seien. Die Angaben der Überlebensrate nach 10 Jahren schwanken zwischen $62 \%$ und $92 \%[5,13,14]$. Bei der systematischen Durchsicht der Ergebnisse fällt auf, dass die exzellenten Resultate häufig nur von den Entwicklern einer Prothese erreicht werden, während andere Autorengruppen über signifikant schlechtere Resultate berichten. Ein Beispiel hierfür ist die Buechel-Pappas-Prothese, die im schwedischen Endoprothesenregister $[13,14]$ signifikant schlechter abschneidet als in den Arbeiten von Buechel et al. [4, 5].

Eine sehr umfassende Darstellung der Komplikationsraten und Ergebnisse lie- ferten Coetzee u. Hurwitz [6]. In ihrem Review über 2386 Prothesen der 2. Generation berichteten sie über eine Gesamtkomplikationsrate von $29,5 \%$ bei einer Überlebensrate nach 10 Jahren von 87,6\%. Die Hauptprobleme waren Sinterung der Prothese (10,7\%), aseptische Lockerungen $(8,7 \%)$, intraoperative Frakturen $(8,1 \%)$ und Wundheilungsprobleme $(6,6 \%)$.

Ein über lange Zeit unterschätztes Problem ist die Entwicklung von Knochenzysten um die Prothese $[3,12,15]$. Einige Produkte wurden aufgrund dieser Problematik zwischenzeitlich vom Markt genommen. Unklar ist, warum einige $\mathrm{Pa}$ tienten Zysten entwickeln, die so ausgeprägt sein können, dass selbst eine Revisionsoperation mit isolierter Versteifung des oberen Sprunggelenks problematisch sein kann (• Abb. 1). Als Ursache werden Abriebpartikel des Polyethylengleitkerns diskutiert [12]. Möglicherweise spielen aber auch noch andere bisher nicht vollständig verstandene immunologische Prozesse eine Rolle [3]. Von der Zystenbildung besonders betroffen scheinen jüngere Patienten mit hohem Aktivitätsniveau zu sein. Häufig geht es diesen Patienten primär sehr gut, sodass sie in der ersten Phase über ein exzellentes Ergebnis berichten. Da selbst massive Zysten teilweise nicht mit Lockerungsphänomenen einhergehen, fallen sie mitunter als Zufallsbefund im Rahmen von Routinekontrollen auf. Dies ist einer der Gründe, warum die Resultate nach Sprunggelenkendoprothese bei jüngeren und aktiven Menschen signifikant schlechter ausfallen als bei älteren und weniger aktiven Patienten $[16,19]$.

\section{Arthrodese}

Die Arthrodese des oberen Sprunggelenks unterlag in den letzten 10 bis 15 Jahren einem erheblichen Wandel. Viele der Berichte über hohe Raten an persistierenden Beschwerden und Anschlussarthrosen nach Sprunggelenkarthrodese beziehen sich auf Verfahren, die heute in dieser Form nicht mehr durchgeführt werden [1]. Häufig erfolgte eine gleichzeitige Fusion des Subtalargelenks, die ungleich schlechtere funktionelle Ergebnisse liefert. Heute wird, wenn immer vertretbar, das Subtalargelenk erhalten.

Während über lange Zeit in den meisten Fällen die offene Arthrodese mit Schraubenosteosynthese durchgeführt wurde, hat sich zwischenzeitlich die arthroskopische Arthrodese mit minimalem Weichteiltrauma zu einer häufig praktizierten Alternative entwickelt ([22], - Abb. 2, - Abb. 3, • Abb. 4). Im direkten Vergleich von offener mit arthrosko-

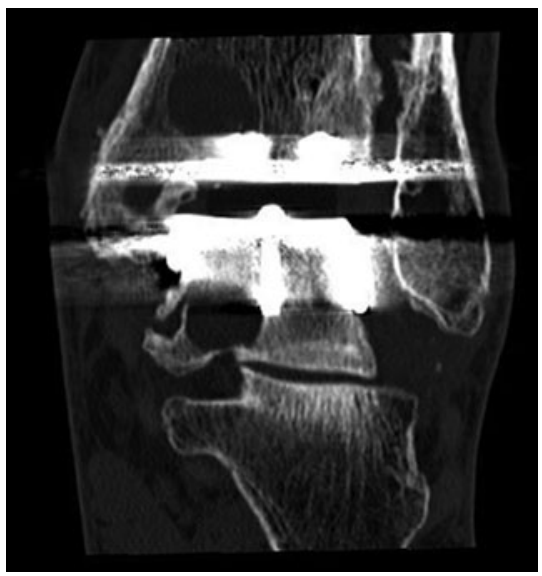

Abb. $1 \Delta$ Ausgedehnte Zystenbildung nach Sprunggelenkendoprothese bei einem 50-jährigen aktiven Patienten 


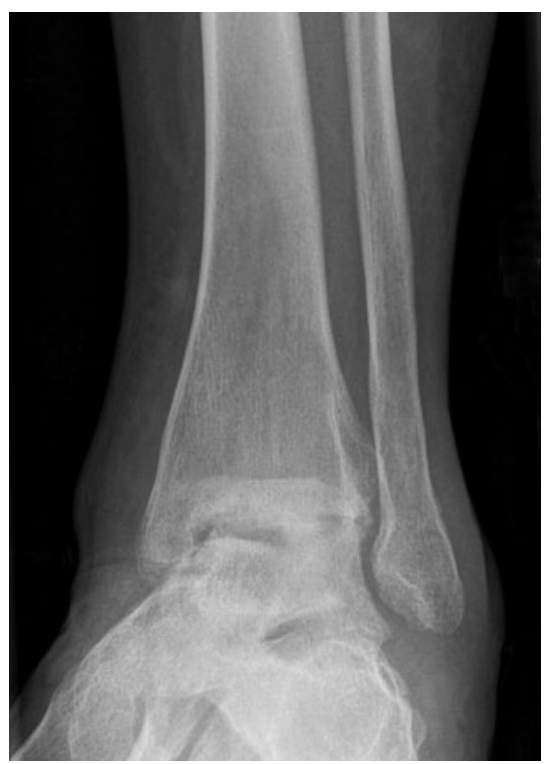

Abb. $2 \Delta$ Arthrose nach Knorpelverletzung, Arthroskopie und Infekt bei einem 35-jährigen Patienten

pischer Arthrodese können im mittelfristigen Verlauf von 1 bis 2 Jahren mit arthroskopischen Techniken bessere Ergebnisse erreicht werden [21]. Der wesentliche Vorteil liegt im minimalen Weichteiltrauma.

Die Grenzen des arthroskopischen Verfahrens sind Achsfehlstellungen von über 15-20\%, wobei im Einzelfall das Korrekturpotenzial auch von der Erfahrung des Operateurs abhängig ist. Dannawi et al. [8] beobachteten in ihrer Studie von 2011 bei ausgeprägten Achsfehlstellungen zwischen $26^{\circ}$ Valgus und $24^{\circ}$ Varus zwar prolongierte Zeiträume bis zur knöchernen Fusion, konnten nach Abschluss der Konsolidierung aber auch hier überwiegend gute bis sehr gute klinische Ergebnisse nachweisen.

Insgesamt wird bei 75-95\% der operierten Patienten über gute bis sehr gute Ergebnisse berichtet [11, 17, 23]. Die Fähigkeit, selbst Auto zu fahren, erlangen alle Patienten wieder, die dies präoperativ konnten. Moderate Sportarten wie Schwimmen, Radfahren, Golf etc. können $45 \%$ der Patienten postoperativ wieder ausüben [9].

\section{Klinische Ergebnisse Prothese versus Arthrodese}

Eine sehr umfassende Analyse klinischer und radiologischer Ergebnisse stellte die kanadische Forschungsgruppe um Da-

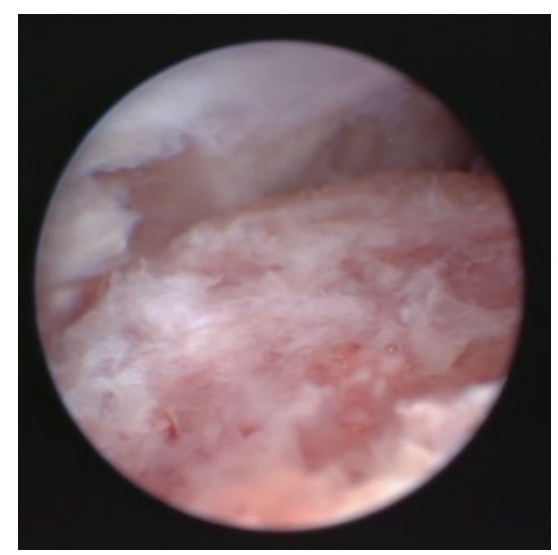

Abb. 3 ॥ Intraoperativer Situs bei arthroskopischer Arthrodese. Der gut durchblutete, spongiöse Knochen des Talus ist gut erkennbar

niels vor [7]. In ihrer prospektiven, therapeutischen Level-II-Studie wurden 281 Patienten mit Sprunggelenkendoprothese und 107 Patienten mit Arthrodese des Sprunggelenks über einen Zeitraum von bis zu 8 Jahren beobachtet. Die Rate schwerer Komplikationen lag in der Gruppe mit Arthrodese bei 7\%, in der mit Endoprothese bei 19\%. Zur Ergebnisbeurteilung wurden die Ankle Osteoarthritis Scale (AOS), der Fragebogen SF-36 sowie verschiedene andere Scores zu Schmerz und Sprunggelenkfunktion verwendet. Bezüglich der Funktion und der Teilhabe am Leben ergaben sich keine Unterschiede zwischen Endoprothese und Arthrodese am Sprunggelenk. Allerdings war die Rate weiterer chirurgischer Maßnahmen und schwerer Komplikationen bei den Patienten mit Endoprothese signifikant höher. Beide Verfahren konnten ihr Potenzial hinsichtlich einer signifikanten Verbesserung der Sprunggelenkfunktion belegen. Trotz des sehr guten Studiendesigns ließ sich aber in keinem der Scores eine funktionelle Überlegenheit der Sprunggelenkprothese objektivieren. Die Rate an operativen Revisionen betrug nach 8 Jahren bei den Prothesen $32 \%$, verglichen mit $7 \%$ nach Arthrodese. Signifikant wird der Unterschied v. a. jenseits einer Beobachtungsdauer von 6 Jahren. Die KaplanMeier-Kurve der kumulativen Inzidenz von Revisionseingriffen in beiden Gruppen legt nahe, dass vergleichende Arbeiten mit einem Follow-up von weniger als 5 Jahren für einen Vergleich der Verfahren wenig Aussagekraft besitzen.

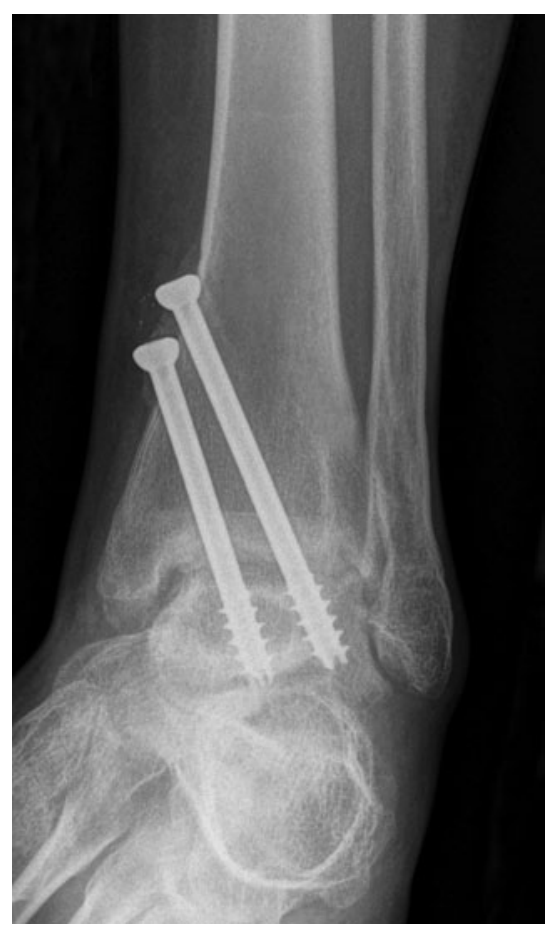

Abb. 4 \ Knöcherne Konsolidierung des oberen Sprunggelenks 12 Wochen nach arthroskopischer Arthrodese

Einige wenige andere Arbeiten beschäftigten sich mit dem direkten Vergleich von Sprunggelenkprothese und Arthrodese. Saltzman et al. [18] berichteten in einer prospektiven, kontrollierten Studie über 446 Patienten mit Sprunggelenkprothese (STAR-Prothese) und $46 \mathrm{~Pa}$ tienten nach Arthrodese. Nach 24 Monaten war der Buechel-Pappas-Funktionsscore bei den Patienten mit Prothese besser als bei den Arthrodesepatienten; die Schmerzscores waren identisch. Slobogean et al. [20] konstatierte $1 \mathrm{Jahr}$ postoperativ ähnliche SF-6D-Werte bei 61 Patienten mit Sprunggelenkprothese und $46 \mathrm{~Pa}$ tienten mit Arthrodese. Limitierend ist allerdings festzuhalten, dass beide Arbeiten einen für die Fragestellung zu kurzen Follow-up aufweisen.

\section{Schlussfolgerungen}

Sowohl die Sprunggelenkendoprothese als auch die Arthrodese des Sprunggelenks sind potente Verfahren zur Behandlung der posttraumatischen Arthrose. Obwohl bei der Arthrodese gezwungenermaßen die Beweglichkeit im oberen Sprunggelenk geopfert wird, schlägt sich dies nicht in signifikant schlechteren 
Funktionsscores im Langzeitverlauf nieder [7]. Eine mögliche Erklärung hierfür ist die bei Beachtung der Achsverhältnisse gute Kompensationsmöglichkeit im Subtalar- und Chopart-Gelenk.

Wird die Arthrodese arthroskopisch durchgeführt, kann das Weichteiltrauma minimiert werden. Ein solches Vorgehen ermöglicht zumindest theoretisch die spätere Revision der Arthrodese in eine Prothese, sollten signifikante Beschwerden durch Anschlussarthrosen auftreten. Auch wenn dies nur in Einzelfällen relevant sein dürfte, bietet allein die Möglichkeit einer Revision in eine Prothese den Patienten eine psychologische Hilfestellung bei der Entscheidung für ein therapeutisches Verfahren.

Die Daten deuten unverändert darauf hin, dass die Belastbarkeit einer Endoprothese am Sprunggelenk signifikant schlechter ist als die einer Arthrodese. Dies schlägt sich in den Ergebnissen bei jüngeren Patienten sowie bei hohem Aktivitätsniveau nieder [16, 19].

Da kein Vorteil im funktionellen Ergebnis bei endoprothetischer Versorgung nachgewiesen werden konnte, erscheint der Preis einer über 30 \%igen Revisionsrate im 10-Jahres-Verlauf für den Erhalt einer gewissen Beweglichkeit im oberen Sprunggelenk hoch [6]. Bei Patienten mit posttraumatischer Arthrose handelt es sich in der Mehrzahl um jüngere, aktive und voll im Arbeitsleben stehende Menschen. Sie weisen auf der Basis der heute zur Verfügung stehenden Daten die schlechtesten Ergebnisse nach Endoprothese auf $[16,19]$. Daher stellt auch heute aus Sicht der Autoren die Arthrodese des oberen Sprunggelenks bei der posttraumatischen Arthrose das Therapieverfahren der Wahl dar. Soweit technisch möglich wird hierbei ein arthroskopisches Vorgehen bevorzugt [22]. Diese Aussage sollte allerdings nicht dahingehend interpretiert werden, dass in Einzelfällen unter Berücksichtigung von Begleiterkrankungen oder Komorbiditäten (z. B. chronische Polyarthritis, degenerative Schäden an anderen Gelenken) die individuelle Entscheidung auch zugunsten einer Endoprothese fallen kann.

Trauma Berufskrankh 2016 · [Suppl 3]: 18:S222-S225 DOI 10.1007/s10039-015-0069-6

(c) Springer-Verlag Berlin Heidelberg 2015

\section{Walther · A. Röser}

\section{Therapie der posttraumatischen Arthrose am Sprunggelenk. Argumente für eine Arthrodese}

\section{Zusammenfassung}

Hintergrund. Trotz der nun mehrere Jahrzehnte umfassenden Erfahrung mit der endoprothetischen Versorgung des Sprunggelenks ließ sich die Überlegenheit der Prothese bisher nicht belegen.

Endoprothese. Bei ähnlichen funktionellen Resultaten im Langzeitverlauf liegt die Revisionsrate 10 Jahre nach endoprothetischer Versorgung bei ca. $30 \%$. Ungelöste Probleme der Endoprothese sind die aseptische Lockerung, Zystenbildung, Low-Grade-Infekte und heterotope Ossifikationen. Verschiedene Arbeiten haben signifikant schlechtere Ergebnisse nach endoprothetischer Versorgung jüngerer und aktiver Menschen nachgewiesen.

Arthrodese. Demgegenüber haben die Operationstechniken für die Arthrodese des Sprunggelenks einen großen Entwicklungsschub erfahren. Solange keine ausgedehnten Knochendefekte oder schwere Achsfehlstellungen vorliegen, kann die Arthrodese heute in vielen Fällen arthroskopisch mit minima- lem Weichteiltrauma durchgeführt werden. Dies bietet insbesondere auch Vorteile bei vorbestehenden ausgedehnten Weichteilvernarbungen, die bei posttraumatischen Arthrosen nicht selten sind. Die Anschlussarthrosen sind v. a. ein Problem bei unzureichender Beachtung der Achsstellung.

Ergebnisse. Die Mehrzahl der Patienten mit posttraumatischer Arthrose ist jünger und durch ein hohes Aktivitätsniveau gekennzeichnet. Speziell für diese Patienten stellt die Arthrodese des oberen Sprunggelenks ein zuverlässiges und hinsichtlich der Langzeitergebnisse stabiles Therapieverfahren dar. Für die Arthrodese sprechen insbesondere die im Vergleich zur Endoprothese signifikant geringere Revisionsrate sowie die seltenen schweren Komplikationen bei funktionell identischen Langzeitergebnissen.

Schlüsselwörter

Endoprothese - Sprunggelenk · Arthrodese . Trauma · Arthrose

\section{Therapy of posttraumatic arthritis of the ankle. Arguments for arthrodesis}

\section{Abstract}

Background. After experience of total ankle replacement over more than one decade, there is still no evidence that total ankle replacement is superior to fusion.

Total ankle replacement. Functional results after total ankle replacement and ankle arthrodesis are very similar in the literature; however, the long-term revision rate after total ankle replacement is up to $30 \%$ after 10 years. Unsolved problems after total ankle replacement include aseptic loosening, cyst formation, low-grade infection and heterotopic ossification. There is increasing evidence that the results after total ankle replacement are especially limited in younger and active patients.

Ankle fusion. In contrast, there has been a remarkable development of the surgical techniques for ankle fusion. As long as there are no extensive bone defects or severe malalignment, ankle fusion can be performed arthroscopically with minimal soft tissue trauma. This has special advantages in posttraumatic patients with extensive scar tissue formation. Degenerative arthritis of adjacent joints is an increased problem in patients with persisting hind foot malalignment.

Results. The majority of patients with posttraumatic arthritis are of a younger age and can be characterized by a high activity level. Especially for these patients arthrodesis of the ankle represents a reliable and stable treatment concept, particularly with respect to long-term results. Strong arguments in favor of arthrodesis are the significantly lower revision rate and rare severe complication rate compared to total ankle replacement while providing identical functional longterm results.

\section{Keywords}

Total ankle replacement - Ankle joint . Arthrodesis · Trauma - Degenerative arthritis 


\section{Fazit für die Praxis}

- Das funktionelle Ergebnis beider Verfahren ist vergleichbar.

- Die schlechtesten Ergebnisse nach Endoprothese weisen jüngere, aktive Patienten mit posttraumatischer Arthrose auf.

- Aus Sicht der Autoren stellt auch heute die Arthrodese des oberen Sprunggelenks bei der posttraumatischen Arthrose das Therapieverfahren der Wahl dar, wenn es sich um jüngere, körperlich arbeitende und aktive Menschen handelt.

- Soweit technisch möglich, wird bei der Arthrodese ein arthroskopisches Vorgehen bevorzugt.

- In Einzelfällen kann die individuelle Entscheidung unter Berücksichtigung von Begleiterkrankungen oder Komorbiditäten zugunsten einer Endoprothese fallen.

\section{Korrespondenzadresse}

Prof. Dr. M. Walther
Schön Klinik München
Harlaching, Harlachinger
Straße 51
81547 München
mwalther@schoen-kliniken.de
http://www.schoen-klini-
ken.de

\section{Einhaltung ethischer Richtlinien}

Interessenkonflikt. M. Walther und A. Röser geben an, dass kein Interessenkonflikt besteht.

Dieser Beitrag beinhaltet keine Studien an Menschen oder Tieren.

The supplement containing this article is not sponsored by industry.

\section{Literatur}

1. Ahlberg A, Henricson AS (1981) Late results of ankle fusion. Acta Orthop Scand 52:103-105

2. Barg A, Wimmer MD, Wiewiorski M, Wirtz DC, Pagenstert GI, Valderrabano V (2015) Total ankle replacement. Dtsch Arztebl Int 112:177-184

3. Besse JL (2015) Osteolytic cysts with total ankle replacement: frequency and causes? Foot Ankle Surg 21:75-76

4. Buechel FF Sr, Buechel FF Jr, Pappas MJ (2002) Eighteen-year evaluation of cementless meniscal bearing total ankle replacements. Instr Course Lect 51:143-151
5. Buechel FF Sr, Buechel FF Jr, Pappas MJ (2004) Twenty-year evaluation of cementless mobile-bearing total ankle replacements. Clin Orthop Relat Res 424:19-26

6. Coetzee JC, Hurwitz SR (2010) Arthritis and arthroplasty - the foot and ankle. Saunders, Philadelphia

7. Daniels TR, Younger AS, Penner M, Wing K, Dryden PJ, Wong H, Glazebrook M (2014) Intermediate-term results of total ankle replacement and ankle arthrodesis: a COFAS multicenter study. J Bone Joint Surg Am 96:135-142

8. Dannawi Z, Nawabi DH, Patel A, Leong JJ, Moore DJ (2011) Arthroscopic ankle arthrodesis: are results reproducible irrespective of pre-operative deformity? Foot Ankle Surg 17:294-299

9. Faraj AA, Loveday DT (2014) Functional outcome following an ankle or subtalar arthrodesis in adults. Acta Orthop Belg 80:276-279

10. Glazebrook M, Daniels T, Younger A, Foote CJ, Penner M, Wing K, Lau J, Leighton R, Dunbar M (2008) Comparison of health-related quality of life between patients with end-stage ankle and hip arthrosis. J Bone Joint Surg Am 90:499-505

11. Gougoulias NE, Agathangelidis FG, Parsons SW (2007) Arthroscopic ankle arthrodesis. Foot Ankle Int 28:695-706

12. Harris NJ, Brooke BT, Sturdee S (2009) A wear debris cyst following S.T.A.R. total ankle replacement-surgical management. Foot Ankle Surg 15:43-45

13. Henricson A, Nilsson JA, Carlsson A (2011) 10-year survival of total ankle arthroplasties: a report on 780 cases from the Swedish Ankle Register. Acta Orthop 82:655-659

14. Henricson A, Skoog A, Carlsson A (2007) The Swedish Ankle Arthroplasty Register: an analysis of 531 arthroplasties between 1993 and 2005. Acta Orthop 78:569-574

15. Jensen J, Frokjaer J, Gerke O, Ludvigsen L, Torfing $T$ (2014) Evaluation of periprosthetic bone cysts in patients with a Scandinavian total ankle replacement: weight-bearing conventional digital radiographs versus weight-bearing multiplanar reconstructed fluoroscopic imaging. AJR Am J Roentgenol 203:863-868

16. Kofoed H, Lundberg-Jensen A (1999) Ankle arthroplasty in patients younger and older than 50 years: a prospective series with long-term follow-up. Foot Ankle Int 20:501-506

17. Pierre $A$, Hulet $C$, Locker $B$, Souquet $D$, Jambou $S$, Vielpeau C (2003) [Arthroscopic tibio-talar arthrodesis: limitations and indications in 20 patients]. Rev Chir Orthop Reparatrice Appar Mot 89:144151

18. Saltzman CL, Mann RA, Ahrens JE, Amendola A, Anderson RB, Berlet GC, Brodsky JW, Chou LB, Clanton TO, Deland JT, Deorio JK, Horton GA, Lee TH, Mann JA, Nunley JA, Thordarson DB, Walling AK, Wapner KL, Coughlin MJ (2009) Prospective controlled trial of STAR total ankle replacement versus ankle fusion: initial results. Foot Ankle Int 30:579-596

19. Schenk K, Lieske S, John M, Franke K, Mouly S, Lizee E, Neumann W (2011) Prospective study of a cementless, mobile-bearing, third generation total ankle prosthesis. Foot Ankle Int 32:755-763

20. Slobogean GP, Younger A, Apostle KL, Marra CA Wing K, Penner M, Daniels T, Glazebrook M (2010) Preference-based quality of life of end-stage ankle arthritis treated with arthroplasty or arthrodesis. Foot Ankle Int 31:563-566
21. Townshend D, Di SM, Krause F, Penner M, Younger A, Glazebrook M, Wing K (2013) Arthroscopic versus open ankle arthrodesis: a multicenter comparative case series. J Bone Joint Surg Am 95:98-102

22. Winson IG, Robinson DE, Allen PE (2005) Arthroscopic ankle arthrodesis. J Bone Joint Surg Br 87:343-347

23. Zvijac JE, Lemak L, Schurhoff MR, Hechtman KS, Uribe JW (2002) Analysis of arthroscopically assisted ankle arthrodesis. Arthroscopy 18:70-75 